\title{
Alcohol is not associated with increased mortality in adolescent traumatic brain injury patients
}

\author{
Zachary N. Lu ${ }^{1} \cdot$ Eric O. Yeates ${ }^{1} \cdot$ Areg Grigorian $^{2} \cdot$ Russell G. Algeo $^{1} \cdot$ Catherine M. Kuza $^{3} \cdot$ Theresa L. Chin $^{1}$. \\ Megan Donnelly ${ }^{1} \cdot$ Allen Kong $^{1} \cdot$ Jeffry Nahmias ${ }^{1}$ (i)
}

Accepted: 13 December 2021 / Published online: 27 December 2021

(c) The Author(s) 2021

\begin{abstract}
Purpose Compared to adults, there is a paucity of data regarding the association of a positive alcohol screen (PAS) and outcomes in adolescent patients with traumatic brain injury (TBI). We hypothesize adolescent TBI patients with a PAS on admission to have increased mortality compared to patients with a negative alcohol screen.

Methods The 2017 Trauma Quality Improvement Program database was queried for patients aged 13-17 years presenting with a TBI and serum alcohol screen. Patients with missing information regarding midline shift on imaging and Glasgow Coma Scale (GCS) score were excluded. A multivariable logistic regression analysis for mortality was performed.

Results From 2553 adolescent TBI patients with an alcohol screen, 220 (8.6\%) had a PAS. Median injury severity scores and rates of penetrating trauma (all $p>0.05$ ) were similar between alcohol positive and negative patients. Patients with a PAS had a similar mortality rate $(13.2 \%$ vs. $12.1 \%, p=0.64)$ compared to patients with a negative screen. Multivariate logistic regression controlling for risk factors associated with mortality revealed a PAS to confer a similar risk of mortality compared to alcohol negative patients $(p=0.40)$.

Conclusion Adolescent TBI patients with a PAS had similar associated risk of mortality compared to patients with a negative alcohol screen.
\end{abstract}

Keywords Adolescent $\cdot$ Traumatic brain injury $\cdot$ Alcohol $\cdot$ Mortality $\cdot$ Outcomes $\cdot$ Database

\section{Introduction}

Traumatic brain injuries (TBI) are common among adolescents and result in over 1 billion dollars in hospital-related costs annually, as well as substantial morbidity and mortality
$[1,2]$. Alcohol use is identified in up to $5 \%$ of traumatically injured adolescent patients [3]. Understanding the impact of alcohol in adolescents suffering from TBI may be useful in predicting outcomes in this population.

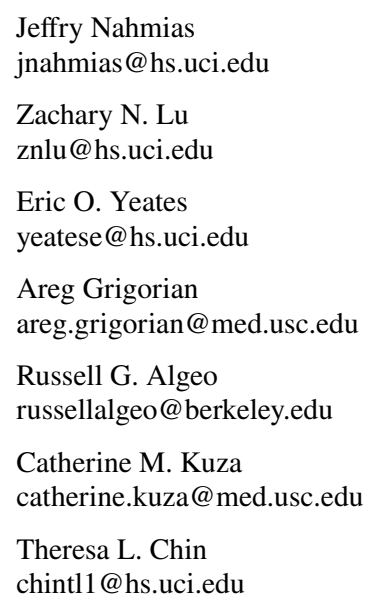

\section{Megan Donnelly \\ mrdonnel@hs.uci.edu}

Allen Kong

konga@hs.uci.edu

1 Division of Trauma, Burns and Surgical Critical Care, Department of Surgery, University of California, Irvine, 333 City Blvd. West, Suite 1600, Orange, CA 92868-3298, USA

2 Department of Surgery, University of Southern California (USC), 1520 San Pablo St., Suite 4300, Los Angeles, CA 90033, USA

3 Department of Anesthesiology, Keck School of Medicine, University of Southern California, 1250 San Pablo St., Suite 3600, Los Angeles, CA 90033, USA 
Alcohol's impact on mortality and other outcomes in adult TBI has been extensively studied. Existing studies have had mixed results, with some reporting lower mortality in alcohol positive patients [4-8], while others demonstrating similar or higher mortality compared to alcohol negative patients [6, 9-15]. Reported results on the effects of other outcomes including hospital length of stay (LOS), intensive care unit (ICU) LOS, and ventilator days between alcohol positive and negative patients are also variable $[4,5,7,9$, $11,12,14,16,17]$. A single national database study in adolescent trauma patients showed higher risk of mortality and hospital complications including pneumonia and sepsis for patients with a positive alcohol screen, but did not specifically focus on patients with traumatic brain injury [18].

This study aimed to investigate the effects of alcohol on adolescent patients sustaining TBI, hypothesizing that adolescent TBI patients with positive alcohol screens on admission would have increased mortality, complications, and LOS compared to adolescent TBI patients with negative alcohol screens.

\section{Materials and methods}

The 2017 Trauma Quality Improvement Program (TQIP) database was queried for all patients between the ages of 13 and 17 years old who sustained a TBI (utilizing ICD10 codes 800-801.96, 803-804.99, 850-854.19) and had a documented alcohol screen on admission. The TQIP database includes data collected from over 700 trauma centers from across the United States. Data quality and accuracy is frequently assessed and optimized via validation programs that measure errors and identify portions of the database that require further assessment [19]. Patients with missing information regarding the presence/absence of midline shift on head computed tomography (CT) imaging or Glasgow Coma Scale (GCS) score on admission were excluded. Midline shift was defined in TQIP as $>5 \mathrm{~mm}$ shift of the brain past its center line within 24-h after time of injury. Patients were placed into two groups, patients with a positive alcohol screen and patients with a negative alcohol screen. The use of this database was approved by the Institutional Review Board of the University of California, Irvine and deemed exempt from need for written informed consent as it utilized a deidentified national database. This manuscript was prepared using the pertinent Strengthening the Reporting of Observational Epidemiology (STROBE) Statement guidelines.

The primary outcome was mortality. The secondary outcomes included hospital LOS, ICU LOS, ventilator days, and complications including unplanned ICU admission, unplanned intubation, unplanned return to the operating room, pneumonia or ventilator-associated pneumonia
(VAP), organ space surgical site infection (SSI), superficial SSI, catheter-associated urinary tract infection (CAUTI), central line-associated bloodstream infection (CLABSI), sepsis, deep vein thrombosis (DVT), pulmonary embolism, and acute kidney injury. Demographics collected included sex and age. Comorbidities collected included diabetes and smoking. The American College of Surgeons (ACS) trauma center level (i.e., Level-I vs Level-II) was also collected. Mechanism of injuries included motor vehicle collisions (MVC), bicycle collisions, falls, gunshot wounds, and stab wounds. Data on presentation including hypotension on arrival (defined as systolic blood pressure (SBP) $<90 \mathrm{mmHg}$ ), tachypnea on arrival (respiratory rate $>22$ breaths/min), tachycardia on arrival (defined as heart rate $>120$ beats/min), SBP on arrival, respiratory rate on arrival, and heart rate on arrival were collected. Injury data included GCS score on admission, injury severity score (ISS), and abbreviated injury scale (AIS) $>3$ for the head, spine, thorax, and abdomen. Patients who tested positive for alcohol were compared to patients who tested negative using Chi-squared tests for categorical variables and Mann-Whitney $U$ tests for continuous variables. Patients aged 13-15 years old were also compared to those 16-17 years old.

Next, univariate analysis for risk of mortality was performed using risk factors determined based on author consensus and a review of the literature, which included severe AIS - head (grade $>3$ ), GCS $\leq 8$, presence of midline shift, hypotension, tachycardia, and smoking [4, 19-21]. Next, variables with a moderate correlation with mortality $(p \leq 0.2)$ were included in a multiple logistic regression model to identify independent risk factors for mortality. A blood alcohol concentration (BAC) of greater than $80 \mathrm{mg} /$ dL was compared to concentrations lower than $80 \mathrm{mg} / \mathrm{dL}$ in patients with a positive alcohol screen. $p$ values were considered statistically significant if $<0.05$. The process was repeated for patients aged 13-15 years old and those aged 16-17 years old. This analysis was performed with IBM SPSS Statistics for Windows (Version 24, IBM Corp., Armonk, NY).

\section{Results}

\section{Demographics, comorbidities, and injury characteristics}

A total of 2553 adolescent TBI patients were identified, and $220(8.6 \%)$ tested positive for alcohol. Compared to patients who screened negative for alcohol, patients who screened positive for alcohol were statistically older (15.9 vs. 15.6 years, $p=0.001$ ) and had higher rates of smoking $(10.0 \%$ vs. $5.4 \%, p=0.005)$, but were otherwise similar 
with regards to sex and comorbidities (all $p>0.05$ ). ISS and AIS for the head, spine, thorax, and abdomen were similar between the two groups (all $p>0.05$ ). Alcohol positive patients had higher rates of stab wounds $(1.4 \%$ vs. $0.3 \%$, $p=0.008)$ and MVC (58.6\% vs. $50.2 \%, p=0.017)$, but lower rates of bicycle collisions (6.3\% vs. $2.7 \%, p=0.033$ ). Compared to alcohol negative patients, alcohol positive patients more commonly had a GCS of $\leq 8$ on admission $(45.4 \%$ vs. $38.1 \%, p=0.036)$ and had a statistically higher, lowest median heart rate on arrival ( 98.5 vs. 93.0 beats $/ \mathrm{min}$, $p=0.016$ ). The two cohorts were otherwise similar with regards to vital signs on arrival (all $p>0.05$ ) (Table 1). Compared to patients aged 13-15, patients aged 16-17 had higher rates of diabetes ( $1.0 \%$ vs. $0.2 \%, p=0.017)$, hypertension $(0.7 \%$ vs. $0.1 \%, p=0.036)$, and smoking $(8.5 \%$ vs. $1.8 \%, p<0.001)$. In addition, $16-17$ year old patients had higher rates of presenting from a MVC $(55.8 \%$ vs. $43.6 \%$, $p<0.001$ ), while 13-15 year old patients had higher rates of presenting as bicycle collisions $(9.6 \%$ vs. $3.6 \%, p<0.001)$, with tachypnea on arrival $(20.7 \%$ vs. $16.5 \%, p=0.007)$, lower median arrival SBP (128 vs. $130, p=0.05)$, and higher median arrival heart rate (96 vs. 93, $p=0.018$ ). Rates of presentation to Level-I trauma centers did not differ between alcohol positive and negative patients nor patients aged $13-15$ and $16-17$ (Table 2).

\section{Outcomes}

Alcohol positive adolescent trauma patients had a similar mortality rate compared to patients with a negative alcohol screen $(13.2 \%$ vs. $12.1 \%, p=0.64)$. Additionally, there were no differences in hospital LOS (4.0 vs. 4.0 days, $p=0.80)$ and ICU LOS (3.0 vs. 3.0 days, $p=0.40$ ) between cohorts. Both groups also had similar ventilator days and complications (all $p>0.05$ ) (Table 3). Patients aged 13-15 had a shorter hospital LOS (4.0 vs. 5.0, $p=0.002$ ), but similar mortality, ICU LOS, ventilator days, and rates of
Table 1 Demographics, comorbidities, and injury characteristics of adolescent traumatic brain injury patients with and without positive alcohol screens

\begin{tabular}{|c|c|c|c|}
\hline Characteristic & $\begin{array}{l}\text { Negative alcohol screen } \\
(n=2333)\end{array}$ & $\begin{array}{l}\text { Positive alcohol } \\
\text { screen }(n=220)\end{array}$ & $p$ value \\
\hline Age, years, mean \pm SD & $15.6 \pm 1.3$ & $15.9 \pm 1.3$ & 0.001 \\
\hline Male, $n(\%)$ & $1692(72.5 \%)$ & $152(69.1 \%)$ & 0.277 \\
\hline \multicolumn{4}{|l|}{ Comorbidities, $n(\%)$} \\
\hline Diabetes & $14(0.6 \%)$ & $3(1.4 \%)$ & 0.183 \\
\hline Smoking & $126(5.4 \%)$ & $22(10.0 \%)$ & 0.005 \\
\hline \multicolumn{4}{|l|}{ Mechanism of injury, $n(\%)$} \\
\hline Motor vehicle collision & $1172(50.2 \%)$ & $129(58.6 \%)$ & $\mathbf{0 . 0 1 7}$ \\
\hline Bicycle collision & $147(6.3 \%)$ & $6(2.7 \%)$ & $\mathbf{0 . 0 3 3}$ \\
\hline Fall & $312(13.4 \%)$ & $30(13.6 \%)$ & 0.913 \\
\hline Gunshot wound & $194(8.3 \%)$ & $15(6.8 \%)$ & 0.439 \\
\hline Stab wound & $6(0.3 \%)$ & $3(1.4 \%)$ & 0.008 \\
\hline Level-I trauma center, $n(\%)$ & $1218(52.5 \%)$ & $101(45.9 \%)$ & 0.074 \\
\hline Hypotensive on arrival, $n(\%)$ & $109(4.7 \%)$ & $7(3.2 \%)$ & 0.310 \\
\hline Tachypneic on arrival, $n(\%)$ & $422(18.1 \%)$ & $43(19.5 \%)$ & 0.592 \\
\hline Tachycardic on arrival, $n(\%)$ & $383(16.4 \%)$ & $37(16.8 \%)$ & 0.878 \\
\hline SBP on arrival, median (IQR) & $129.0(25)$ & $128.0(28)$ & 0.474 \\
\hline Respiratory rate on arrival, median (IQR) & $18.0(5)$ & $18(6)$ & 0.792 \\
\hline Heart rate on arrival, median (IQR) & $93.0(33)$ & $98.5(32)$ & 0.016 \\
\hline GCS $\leq 8$ on admission, $n(\%)$ & $879(38.1 \%)$ & $98(45.4 \%)$ & $\mathbf{0 . 0 3 6}$ \\
\hline ISS, median (IQR) & $17.0(17)$ & $17.0(16)$ & 0.336 \\
\hline \multicolumn{4}{|l|}{ AIS (grade $>3), n(\%)$} \\
\hline Head & $1079(46.2 \%)$ & $102(46.4 \%)$ & 0.974 \\
\hline Spine & $26(1.1 \%)$ & $3(1.4 \%)$ & 0.739 \\
\hline Thorax & $124(5.3 \%)$ & $8(3.6 \%)$ & 0.282 \\
\hline Abdomen & $93(4.0 \%)$ & $6(2.7 \%)$ & 0.355 \\
\hline
\end{tabular}

A bolded row denotes a variable with $p<0.05$, SD standard deviation, ISS injury severity score, IQR interquartile range, $S B P$ systolic blood pressure, $C O P D$ chronic obstructive pulmonary disease, AIS abbreviated injury scale, CVA cerebrovascular accident, GCS Glasgow Coma Scale, hypotension is defined as $<90 \mathrm{mmHg}$, tachypnea is defined as respiratory rate $>22$ breaths/min, tachycardia is defined as heart rate $>120$ beats/min 
Table 2 Demographics, comorbidities, and injury characteristics of adolescent traumatic brain injury patients compared by age

\begin{tabular}{|c|c|c|c|}
\hline Characteristic & Ages $13-15(n=1022)$ & Ages $16-17(n=1531)$ & $p$ value \\
\hline Age, years, mean \pm SD & $14.3 \pm 0.8$ & $16.6 \pm 0.5$ & $<0.001$ \\
\hline Male, $n(\%)$ & $739(72.3 \%)$ & $1105(72.2 \%)$ & 0.941 \\
\hline \multicolumn{4}{|l|}{ Comorbidities, $n(\%)$} \\
\hline COPD & $8(0.8 \%)$ & $7(0.5 \%)$ & 0.292 \\
\hline History of CVA & $1(0.1 \%)$ & $2(0.1 \%)$ & 0.813 \\
\hline Diabetes & $2(0.2 \%)$ & $15(1.0 \%)$ & 0.017 \\
\hline Hypertension & $1(0.1 \%)$ & $10(0.7 \%)$ & 0.036 \\
\hline Smoking & $18(1.8 \%)$ & $130(8.5 \%)$ & $<0.001$ \\
\hline \multicolumn{4}{|l|}{ Mechanism of injury, $n(\%)$} \\
\hline Motor vehicle collision & $446(43.6 \%)$ & $855(55.8 \%)$ & $<0.001$ \\
\hline Bicycle collision & $98(9.6 \%)$ & $55(3.6 \%)$ & $<0.001$ \\
\hline Fall & $138(13.5 \%)$ & $204(13.3 \%)$ & 0.897 \\
\hline Gunshot wound & $81(7.9 \%)$ & $128(8.4 \%)$ & 0.695 \\
\hline Stab wound & $2(0.2 \%)$ & $7(0.5 \%)$ & 0.275 \\
\hline Level-I trauma center, $n(\%)$ & $520(50.9 \%)$ & $799(52.2 \%)$ & 0.517 \\
\hline Hypotensive on arrival, $n(\%)$ & $48(4.7 \%)$ & $68(4.4 \%)$ & 0.762 \\
\hline Tachypneic on arrival, $n(\%)$ & $212(20.7 \%)$ & $253(16.5 \%)$ & 0.007 \\
\hline Tachycardic on arrival, $n(\%)$ & $168(16.4 \%)$ & $252(16.5 \%)$ & 0.989 \\
\hline SBP on arrival, median (IQR) & $128(25)$ & $130(25)$ & 0.005 \\
\hline Respiratory rate on arrival, median (IQR) & $18(6)$ & $18(5)$ & 0.472 \\
\hline Heart rate on arrival, median (IQR) & $96(31)$ & $93(34)$ & 0.018 \\
\hline GCS $\leq 8$ on admission, $n(\%)$ & $404(40.1 \%)$ & $573(37.8 \%)$ & 0.254 \\
\hline ISS, median (IQR) & $17(16)$ & $17(19)$ & 0.143 \\
\hline \multicolumn{4}{|l|}{ AIS (grade $>3), n(\%)$} \\
\hline Head & $453(44.3 \%)$ & $728(47.6 \%)$ & 0.109 \\
\hline Spine & $7(0.7 \%)$ & $22(1.4 \%)$ & 0.079 \\
\hline Thorax & $49(4.8 \%)$ & $83(5.4 \%)$ & 0.483 \\
\hline Abdomen & $34(3.3 \%)$ & $65(4.2 \%)$ & 0.239 \\
\hline
\end{tabular}

A bolded row denotes a variable with $p<0.05$, SD standard deviation, ISS injury severity score, IQR interquartile range, $S B P$ systolic blood pressure, COPD chronic obstructive pulmonary disease, AIS abbreviated injury scale, CVA cerebrovascular accident, GCS Glasgow Coma Scale, hypotension is defined as $<90 \mathrm{mmHg}$, tachypnea is defined as respiratory rate $>22$ breaths/min, tachycardia is defined as heart rate $>120$ beats $/ \mathrm{min}$

complications compared to patients aged 16-17 (all $p>0.05)$ (Table 4).

\section{Univariate analysis for risk of mortality}

Univariate analysis showed that a severe AIS-head (OR 11.65, CI 8.11-16.73, $p<0.001)$, severe AIS-thorax (OR 3.18, CI 2.14-4.73, $p<0.001$ ), severe AIS-abdomen (OR 2.42, CI 1.50-3.89, $p<0.001$ ), midline shift (OR 6.06, CI 4.65-7.91, $p<0.001$ ), hypotension (OR 12.29, CI 8.31-18.18, $p<0.001$ ), tachycardia (OR 3.93, CI 3.03-5.09, $p<0.001$ ), and smoking (OR 0.51, CI 0.26-0.97, $p=0.041$ ) were associated risk factors for mortality. A positive alcohol screen (OR 1.104, CI 0.73-1.66, $p=0.635$ ), severe AISspine (OR 2.32, CI 0.98-5.49, $p=0.054$ ), and tachypnea (OR 1.11, CI 0.82-1.50, $p=0.495$ ) were not associated with mortality risk (Table 5).

\section{Multivariable logistic regression analysis for risk of mortality}

\section{All patients}

Multivariate analysis demonstrated that severe AIS-head (OR 4.94, CI 3.26-7.50, $p<0.001$ ), GCS $\leq 8$ at presentation (OR 33.31, CI 16.81-66.02, $p<0.001$ ), midline shift (OR 2.76, CI 2.00-3.83, $p<0.001$ ), hypotension (OR 6.99, CI 4.25-11.50, $p<0.001$ ), and tachycardia (OR 2.18, CI 1.57-3.30, $p<0.001)$ were associated risk factors for mortality. Smoking was associated with a decreased risk for mortality (OR 0.44 , CI $0.20-0.97, p=0.041$ ). A positive alcohol screen was not an independent associated risk factor for mortality (OR 1.24, CI 0.75-2.07, $p=0.402$ ) (Table 6). 
Table 3 Clinical outcomes in adolescent traumatic brain injury patients with and without positive alcohol screens
Table 4 Clinical outcomes in adolescent traumatic brain injury patients compared by age

\begin{tabular}{llll}
\hline Outcome & $\begin{array}{l}\text { Negative alcohol screen } \\
(n=2333)\end{array}$ & $\begin{array}{l}\text { Positive alcohol screen } \\
(n=220)\end{array}$ & $p$ value \\
\hline LOS, days, median (IQR) & $4(8)$ & $4(8)$ & 0.800 \\
ICU LOS, days, median (IQR) & $3(5)$ & $3(6)$ & 0.400 \\
Ventilator days, median (IQR) & $3(6)$ & $2(6)$ & 0.084 \\
Complications, $n(\%)$ & & & \\
Unplanned ICU admission & $23(1.0 \%)$ & $4(1.8 \%)$ & 0.249 \\
Unplanned intubation & $26(1.1 \%)$ & $1(0.5 \%)$ & 0.360 \\
Unplanned return to OR & $20(0.9 \%)$ & $3(1.4 \%)$ & 0.447 \\
Pneumonia/VAP & $55(2.4 \%)$ & $3(1.4 \%)$ & 0.344 \\
Organ space SSI & $2(0.1 \%)$ & $0(0.0 \%)$ & 0.664 \\
Superficial incision SSI & $8(0.3 \%)$ & $0(0.0 \%)$ & 0.384 \\
CAUTI & $18(0.8 \%)$ & $0(0.0 \%)$ & 0.191 \\
CLABSI & $7(0.3 \%)$ & $1(0.5 \%)$ & 0.695 \\
Sepsis & $8(0.3 \%)$ & $1(0.5 \%)$ & 0.789 \\
DVT & $18(0.8 \%)$ & $1(0.5 \%)$ & 0.601 \\
Pulmonary embolism & $7(0.3 \%)$ & $0(0.0 \%)$ & 0.416 \\
Kidney injury & $12(0.5 \%)$ & $1(0.5 \%)$ & 0.905 \\
Mortality, $n(\%)$ & $282(12.1 \%)$ & $29(13.2 \%)$ & 0.635 \\
\hline
\end{tabular}

$L O S$ length of stay, $I Q R$ interquartile range, $I C U$ intensive care unit, $O R$ operating room, SSI surgical site infection, VAP ventilator-associated pneumonia, CAUTI catheter-associated urinary tract infection, CLABSI central line-associated bloodstream infection, $D V T$ deep vein thrombosis

\begin{tabular}{llll}
\hline Outcome & Ages 13-15 $(n=1022)$ & Ages 16-17 $(n=1531)$ & $p$ value \\
\hline LOS, days, median (IQR) & $\mathbf{4}(\mathbf{7})$ & $\mathbf{5}(\mathbf{9})$ & $\mathbf{0 . 0 0 2}$ \\
ICU LOS, days, median (IQR) & $3(5)$ & $3(6)$ & 0.530 \\
Ventilator days, median (IQR) & $3(5)$ & $3(6)$ & 0.373 \\
Complications, $n$ (\%) & & & \\
Unplanned ICU admission & $8(0.8 \%)$ & $19(1.2 \%)$ & 0.267 \\
Unplanned intubation & $13(1.3 \%)$ & $14(0.9 \%)$ & 0.387 \\
Unplanned return to OR & $7(0.7 \%)$ & $16(1.0 \%)$ & 0.345 \\
Pneumonia/VAP & $18(1.8 \%)$ & $40(2.6 \%)$ & 0.157 \\
Organ space SSI & $0(0.0 \%)$ & $2(0.1 \%)$ & 0.248 \\
Superficial incision SSI & $2(0.2 \%)$ & $6(0.4 \%)$ & 0.385 \\
CAUTI & $6(0.6 \%)$ & $12(0.8 \%)$ & 0.561 \\
CLABSI & $4(0.4 \%)$ & $4(0.3 \%)$ & 0.564 \\
Sepsis & $2(0.2 \%)$ & $7(0.5 \%)$ & 0.275 \\
DVT & $7(0.7 \%)$ & $12(0.8 \%)$ & 0.776 \\
Pulmonary embolism & $2(0.2 \%)$ & $5(0.3 \%)$ & 0.535 \\
Kidney injury & $8(0.8 \%)$ & $5(0.3 \%)$ & 0.113 \\
Mortality, $n$ (\%) & $115(11.3 \%)$ & $196(12.8 \%)$ & 0.241 \\
\hline
\end{tabular}

A bolded row denotes a variable with $p<0.05$, LOS length of stay, IQR interquartile range, ICU intensive care unit, $O R$ operating room, $C V A$ cerebrovascular accident, SSI surgical site infection, VAP ventilatorassociated pneumonia, CAUTI catheter-associated urinary tract infection, CLABSI central line-associated bloodstream infection, $D V T$ deep vein thrombosis 
Table 5 Univariate analysis for risk of mortality in adolescent traumatic brain injury patients with and without positive alcohol screens
Table 6 Multivariable logistic regression analysis for risk of mortality in adolescent traumatic brain injury patients with and without positive alcohol screens

\begin{tabular}{lrlr}
\hline Risk factor & \multicolumn{1}{l}{ OR } & CI & $p$ value \\
\hline Positive alcohol screen & 1.104 & $0.733-1.664$ & 0.635 \\
Severe AIS-head $($ grade $>$ 3) & $\mathbf{1 1 . 6 5 0}$ & $\mathbf{8 . 1 1 4 - 1 6 . 7 2 6}$ & $<\mathbf{0 . 0 0 1}$ \\
Severe AIS-spine $($ grade $>$ 3) & 2.324 & $0.984-5.485$ & 0.054 \\
Severe AIS-thorax $($ grade $>$ 3) & $\mathbf{3 . 1 8 1}$ & $\mathbf{2 . 1 3 8 - 4 . 7 3 2}$ & $<\mathbf{0 . 0 0 1}$ \\
Severe AIS-abdomen $($ grade $>$ 3) & $\mathbf{2 . 4 1 6}$ & $\mathbf{1 . 5 0 1 - 3 . 8 8 9}$ & $<\mathbf{0 . 0 0 1}$ \\
Presence of midline shift on CT scan head & $\mathbf{6 . 0 6 4}$ & $\mathbf{4 . 6 4 7 - 7 . 9 1 3}$ & $<\mathbf{0 . 0 0 1}$ \\
Hypotension & $\mathbf{1 2 . 2 8 9}$ & $\mathbf{8 . 3 0 9 - 1 8 . 1 7 7}$ & $<\mathbf{0 . 0 0 1}$ \\
Tachypnea & 1.110 & $0.822-1.498$ & 0.495 \\
Tachycardia & $\mathbf{3 . 9 2 8}$ & $\mathbf{3 . 0 3 1 - 5 . 0 8 9}$ & $<\mathbf{0 . 0 0 1}$ \\
Smoking & $\mathbf{0 . 5 0 7}$ & $\mathbf{0 . 2 6 4 - 0 . 9 7 3}$ & $\mathbf{0 . 0 4 1}$ \\
\hline
\end{tabular}

A bolded row denotes a variable with $p<0.05$, AIS abbreviated injury scale, GCS Glasgow Coma Scale, $C T$ computed tomography, hypotension is defined as $<90 \mathrm{mmHg}$, tachypnea is defined as respiratory rate $>22$ breaths $/ \mathrm{min}$, tachycardia is defined as heart rate $>120$ beats $/ \mathrm{min}$

\begin{tabular}{lrlr}
\hline Risk factor & \multicolumn{1}{l}{ OR } & CI & $p$ value \\
\hline Positive alcohol screen & 1.243 & $0.747-2.067$ & 0.402 \\
Severe AIS-head (grade $>$ 3) & $\mathbf{4 . 9 4 3}$ & $\mathbf{3 . 2 5 9 - 7 . 4 9 8}$ & $<\mathbf{0 . 0 0 1}$ \\
GCS $\leq \mathbf{8}$ & $\mathbf{3 3 . 3 1 4}$ & $\mathbf{1 6 . 8 0 9 - 6 6 . 0 2 4}$ & $<\mathbf{0 . 0 0 1}$ \\
Presence of midline shift on CT scan head & $\mathbf{2 . 7 6 4}$ & $\mathbf{1 . 9 9 6 - 3 . 8 2 7}$ & $<\mathbf{0 . 0 0 1}$ \\
Hypotension & $\mathbf{6 . 9 9 4}$ & $\mathbf{4 . 2 5 3 - 1 1 . 5 0 1}$ & $<\mathbf{0 . 0 0 1}$ \\
Tachycardia & $\mathbf{2 . 1 8 2}$ & $\mathbf{1 . 5 6 9 - 3 . 3 0 3 6}$ & $<\mathbf{0 . 0 0 1}$ \\
Smoking & $\mathbf{0 . 4 3 7}$ & $\mathbf{0 . 1 9 8 - 0 . 9 6 6}$ & $\mathbf{0 . 0 4 1}$ \\
\hline
\end{tabular}

A bolded row denotes a variable with $p<0.05$, AIS abbreviated injury scale, GCS Glasgow Coma Scale, $C T$ computed tomography, hypotension is defined as $<90 \mathrm{mmHg}$, tachypnea is defined as respiratory rate $>22$ breaths/min, tachycardia is defined as heart rate $>120$ beats/min
Table 7 Multivariable logistic regression analysis for risk of mortality in adolescent traumatic brain injury patients with a positive alcohol screen

\begin{tabular}{lrll}
\hline Risk factor & \multicolumn{1}{l}{ OR } & CI & $p$ value \\
\hline BAC $>$ 80 $\mathbf{~ m g / d L ~}$ & $\mathbf{0 . 1 9 4}$ & $\mathbf{0 . 0 4 5 - 0 . 8 4 3}$ & $\mathbf{0 . 0 2 9}$ \\
$\begin{array}{l}\text { Severe AIS-head } \\
\quad \text { grade }>\text { 3) }\end{array}$ & $\mathbf{1 0 . 7 7 9}$ & $\mathbf{1 . 8 6 0 - 6 2 . 4 5 3}$ & $\mathbf{0 . 0 0 8}$ \\
GCS $\leq \mathbf{8}$ & $\mathbf{2 2 . 1 6 5}$ & $\mathbf{2 . 5 7 7 - 1 9 0 . 6 1 5}$ & $\mathbf{0 . 0 0 5}$ \\
Presence of midline & $\mathbf{1 2 . 2 9 0}$ & $\mathbf{2 . 9 3 9 - 5 1 . 3 8 4}$ & $\mathbf{0 . 0 0 1}$ \\
$\quad$ shift on CT scan & & & \\
$\quad$ head & & & \\
Hypotension & 7.556 & $0.778-73.410$ & 0.081 \\
Tachycardia & 3.712 & $0.785-17.541$ & 0.098 \\
Smoking & $\mathbf{0 . 0 4 4}$ & $\mathbf{0 . 0 0 2 - 0 . 9 4 9}$ & $\mathbf{0 . 0 4 6}$ \\
\hline
\end{tabular}

A bolded row denotes a variable with $p<0.05$, BAC blood alcohol concentration, $m g$ milligrams, $d L$ deciliter, AIS abbreviated injury scale, GCS Glasgow Coma Scale, CT computed tomography

\section{Positive alcohol screen}

In patients with a positive alcohol screen, $\mathrm{BAC}>80 \mathrm{mg} / \mathrm{dL}$ was associated with a decreased risk for mortality in a multivariable analysis only including patients with a positive alcohol screen (OR 0.194, CI 0.05-0.84, $p=0.029)$ (Table 7).

\section{Ages 13-15 years old}

Multivariate analysis demonstrated that in patients 13-15 years old, severe AIS - head (OR 8.46, CI 3.76-19.02, $p<0.001), \mathrm{GCS} \leq 8$ (OR 83.49, CI 11.41-610.68, $p<0.001$ ), midline shift (OR 2.63, CI 1.49-4.62, $p=0.001$ ), hypotension (OR 8.91, CI 3.98-19.90, $p<0.001$ ), and tachycardia (OR 3.40, CI 1.93-6.01, $p<0.001$ ) were associated risk factors for mortality. A positive alcohol screen (OR 1.04, CI $0.42-2.56, p=0.939)$ and smoking (OR 0.49, CI 0.09-2.65, $p=0.41)$ were not associated with mortality in this age group (Table 8). 
Table 8 Multivariable logistic regression analysis for risk of mortality in adolescent traumatic brain injury patients aged 13-15
Table 9 Multivariable logistic regression analysis for risk of mortality in adolescent traumatic brain injury patients aged 16-17

\begin{tabular}{lrlr}
\hline Risk factor & \multicolumn{1}{l}{ OR } & CI & $p$ value \\
\hline Positive alcohol screen & 1.036 & $0.419-2.561$ & 0.939 \\
Severe AIS-head (grade $>\mathbf{3}$ ) & $\mathbf{8 . 4 5 9}$ & $\mathbf{3 . 7 6 3 - 1 9 . 0 1 7}$ & $<\mathbf{0 . 0 0 1}$ \\
GCS $\leq \mathbf{8}$ & $\mathbf{8 3 . 4 8 9}$ & $\mathbf{1 1 . 4 1 4 - 6 1 0 . 6 8 3}$ & $<\mathbf{0 . 0 0 1}$ \\
Presence of midline shift on CT scan head & $\mathbf{2 . 6 2 6}$ & $\mathbf{1 . 4 9 3 - 4 . 6 1 7}$ & $\mathbf{0 . 0 0 1}$ \\
Hypotension & $\mathbf{8 . 9 0 5}$ & $\mathbf{3 . 9 8 4 - 1 9 . 9 0 2}$ & $<\mathbf{0 . 0 0 1}$ \\
Tachycardia & $\mathbf{3 . 4 0 1}$ & $\mathbf{1 . 9 2 6 - 6 . 0 0 6}$ & $<\mathbf{0 . 0 0 1}$ \\
Smoking & 0.490 & $0.091-2.647$ & 0.407 \\
\hline
\end{tabular}

A bolded row denotes a variable with $p<0.05$, AIS abbreviated injury scale, GCS Glasgow Coma Scale, $C T$ computed tomography

\begin{tabular}{lclr}
\hline Risk factor & \multicolumn{1}{l}{ OR } & CI & $p$ value \\
\hline Positive alcohol screen & 1.346 & $0.717-2.529$ & 0.355 \\
Severe AIS-head (grade $>\mathbf{3}$ ) & $\mathbf{3 . 8 4 3}$ & $\mathbf{2 . 3 4 0 - 6 . 3 1 0}$ & $<\mathbf{0 . 0 0 1}$ \\
GCS $\leq \mathbf{8}$ & $\mathbf{2 7 . 6 5 1}$ & $\mathbf{1 3 . 2 2 0 - 5 7 . 8 3 8}$ & $<\mathbf{0 . 0 0 1}$ \\
Presence of midline shift on CT scan head & $\mathbf{2 . 7 6 4}$ & $\mathbf{1 . 8 4 3 - 4 . 1 4 6}$ & $<\mathbf{0 . 0 0 1}$ \\
Hypotension & $\mathbf{6 . 1 9 6}$ & $\mathbf{3 . 2 3 6}-\mathbf{1 1 . 8 6 2}$ & $<\mathbf{0 . 0 0 1}$ \\
Tachycardia & $\mathbf{1 . 8 0 3}$ & $\mathbf{1 . 1 8 8 - 2 . 7 3 4}$ & $\mathbf{0 . 0 0 6}$ \\
Smoking & $\mathbf{0 . 3 6 2}$ & $\mathbf{0 . 1 4 5 - 0 . 9 0 4}$ & $\mathbf{0 . 0 3 0}$ \\
\hline
\end{tabular}

A bolded row denotes a variable with $p<0.05$, AIS abbreviated injury scale, GCS Glasgow Coma Scale, $C T$ computed tomography

\section{Ages 16-17 years old}

Multivariate analysis in patients aged 16-17 showed that severe AIS-head (OR 3.84, CI 2.34-6.31, $p<0.001$ ), GCS $\leq 8$ (OR 27.65, CI 13.22-57.84, $p<0.001$ ), midline shift (OR 2.76, CI 1.84-4.15, $p<0.001$ ), hypotension (OR 6.20 , CI 3.24-11.86, $p<0.001$ ), tachycardia (OR 1.80, CI $1.19-2.73, p=0.006$ ), and smoking (OR 0.362, CI 0.15-0.90, $p=0.03$ ) were associated risk factors for mortality. A positive alcohol screen (OR 1.35, CI $0.72-2.53, p=0.355$ ) in this age group was not associated with mortality (Table 9).

\section{Discussion}

In contrast to adult TBI patients, there is limited evidence regarding the effects of alcohol on mortality in adolescent TBI patients. This current national database analysis found no difference in rate and associated risk of mortality for alcohol positive compared to alcohol negative adolescent TBI patients. Furthermore, there was no difference in LOS, ventilator days, or hospital complications between the two groups.

Alcohol use is prevalent among the adolescent trauma population and is a significant concern to public health [22]. Alcohol use during adolescence has been shown to increase risk for TBI [23]. However, the effects of alcohol on mortality in the setting of TBI is unknown. This study found that alcohol was not an independent risk factor for mortality in adolescent TBI patients. A national database study on alcohol and adolescent trauma patients found a higher mortality rate in alcohol positive patients [18]. However, it did not focus on TBI patients and did not control for potential confounders for mortality in TBI including ISS, GCS, and hemodynamic instability. Available literature regarding the effects of alcohol on TBI patients focus on the adult population and have mixed results with regards to mortality [4-15, 23]. One of the most robust studies is a meta-analysis by Raj et al., which evaluated 11 studies and over 95,000 patients and reported that alcohol positive patients had lower mortality compared to alcohol negative patients [24]. A purported mechanism behind the potential neuroprotective effect of alcohol is the inhibition of $N$-methyl-D-aspartate receptor (NMDA)-mediated excitotoxicity in TBI and a reduction of deleterious effects of catecholamine surges [24, 25]. In contrast, higher rates of structural brain atrophy and poorer neurologic outcomes have been found in alcohol positive patients [26]. The developing adolescent brain is markedly different compared to the adult brain and, therefore, may respond differently to alcohol in the setting of injury. Alcohol has been shown to be particularly deleterious in the developing adolescent brain [27]. Experimental studies demonstrated adolescent mice with TBI had higher mortality and worse neurologic outcomes compared to adult mice 
[28]. The differences in outcomes between our study and those of prior studies in adults may be explained by developmental changes during the adolescent years. Interestingly, we also found that a blood alcohol concentration of greater than $80 \mathrm{mg} / \mathrm{dL}$ in alcohol positive patients was protective with regards to mortality. This may indicate that there is a dose-dependent relationship that should be explored further. Further prospective basic science and clinical studies are needed to confirm our findings and continue to uncover the full effects of alcohol across all age groups.

Given the lower rate of mortality overall among adolescent trauma patients compared to adults, studying alcohol's effects on other outcomes, such as LOS and complications, may be even more important for the adolescent TBI population. This large database study demonstrated that adolescent TBI patients with positive alcohol screens had similar hospital LOS, ICU LOS, ventilator days, and complications compared to patients who screened negative for alcohol. Outcomes specifically among adolescents with TBI for alcohol positive and negative patients have not been previously compared. Aziz et al. studied outcomes in adolescent trauma and found higher rates of acute respiratory distress syndrome (ARDS), pneumonia, DVT, and pulmonary embolism in alcohol positive adolescents [18]. In contrast, our findings are similar to those of Talving et al. and Scheyerer et al. with adult populations who found no difference in hospital LOS, ICU LOS, and complications between alcohol positive and negative TBI patients $[5,11]$. It is important to recognize the extensively documented effects of alcohol on long-term outcomes including cognition and social performance [27]. The absence of hospital outcome differences in our study highlights the importance of investigating the long-term effects of alcohol on the developing brain. This study is the first to suggest alcohol may not significantly affect hospital complications and LOS among the adolescent TBI population. This could be useful for predicting outcomes and benchmarking quality of care in the adolescent TBI population.

There are multiple limitations to this study including those inherent to a retrospective database study, which includes misclassification and missing variables. In addition, missing pertinent variables from the TQIP database include blood alcohol concentration, CT imaging findings such as a Rotterdam score, concomitant substance use, history of previous TBI, and hospital alcohol screening policies. Data may not include patients who expired prior to presenting to the hospital. While our multivariable analysis attempted to control for many factors, there still may be potential confounders that are not accounted for and should be addressed in future prospective study. Furthermore, a significant limitation is that the TQIP database is confined to index hospitalization data and lacks crucial post-discharge neurologic outcomes $[1,29]$. Specifically cognitive function outcomes such as memory and psychological factors were not available in the database utilized. Finally, as only 220 adolescent patients were alcohol positive, our lack of statistical significance could be the result of limited power.

\section{Conclusions}

This database study demonstrated no difference in rate and associated risk of mortality between alcohol positive and alcohol negative adolescent TBI patients. There was also no difference in LOS or hospital complications between the two groups. The role of alcohol in adolescent TBI is a developing field of research and future basic science studies are needed to elicit the exact impact of alcohol in this population.

Author contributions EY, AG, RA, MD, and JN contributed to the study's concept and design. ZL, EY, AG, RA, CK, TC, MD, AK, and JN contributed to data acquisition and analysis. ZL, EY, and JN contributed to the manuscript drafts. ZL, EY, AG, RA, CK, TC, MD, AK, and $\mathrm{JN}$ contributed to revising the manuscript.

Funding No funds, grants, or other monetary support was received for this study.

\section{Declarations}

Conflict of interest The authors have no financial or non-financial conflicts of interest associated with this study.

Ethics approval The University of California, Irvine institutional review board deemed this study exempt from review given that retrospective data were obtained from a national deidentified database (Trauma Quality Improvement Program, TQIP).

Open Access This article is licensed under a Creative Commons Attribution 4.0 International License, which permits use, sharing, adaptation, distribution and reproduction in any medium or format, as long as you give appropriate credit to the original author(s) and the source, provide a link to the Creative Commons licence, and indicate if changes were made. The images or other third party material in this article are included in the article's Creative Commons licence, unless indicated otherwise in a credit line to the material. If material is not included in the article's Creative Commons licence and your intended use is not permitted by statutory regulation or exceeds the permitted use, you will need to obtain permission directly from the copyright holder. To view a copy of this licence, visit http://creativecommons.org/licenses/by/4.0/.

\section{References}

1. Shaklai S, Peretz R, Spasser R, Simantov M, Groswasser Z (2014) Long-term functional outcome after moderate-to-severe paediatric traumatic brain injury. Brain Inj 28(7):915-921. https://doi.org/ 10.3109/02699052.2013.862739

2. Schneier AJ, Shields BJ, Hostetler SG, Xiang H, Smith GA (2006) Incidence of pediatric traumatic brain injury and associated 
hospital resource utilization in the United States. Pediatrics 118(2):483-492. https://doi.org/10.1542/peds.2005-2588

3. Noh H, Jung KY, Park HS, Cheon YJ (2011) Characteristics of alcohol-related injuries in adolescents visiting the emergency department. J Korean Med Sci 26(3):431-437. https://doi.org/ 10.3346/jkms.2011.26.3.431

4. Chen CM, Yi H-Y, Yoon Y-H, Dong C (2012) Alcohol use at time of injury and survival following traumatic brain injury: results from the National Trauma Data Bank. J Stud Alcohol Drugs 73(4):531-541. https://doi.org/10.15288/jsad.2012.73.531

5. Talving P, Plurad D, Barmparas G et al (2010) Isolated severe traumatic brain injuries: association of blood alcohol levels with the severity of injuries and outcomes. J Trauma 68(2):357-362. https://doi.org/10.1097/TA.0b013e3181bb80bf

6. Tien HCN, Tremblay LN, Rizoli SB et al (2006) Association between alcohol and mortality in patients with severe traumatic head injury. Arch Surg 141(12):1185-1191. https://doi.org/10. 1001/archsurg.141.12.1185

7. Salim A, Teixeira P, Ley EJ, DuBose J, Inaba K, Margulies DR (2009) Serum ethanol levels: predictor of survival after severe traumatic brain injury. J Trauma 67(4):697-703. https://doi.org/ 10.1097/TA.0b013e3181b5dcf2

8. Ward RE, Flynn TC, Miller PW, Blaisdell WF (1982) Effects of ethanol ingestion on the severity and outcome of trauma. Am J Surg 144(1):153-157. https://doi.org/10.1016/0002-9610(82) 90617-1

9. El-Menyar A, Consunji R, Asim M et al (2019) Traumatic brain injury in patients screened for blood alcohol concentration based on the mechanism of injury. Brain Inj 33(4):419-426. https://doi. org/10.1080/02699052.2018.1553065

10. Rao AJ, Lin AL, Hilliard C et al (2018) Blood ethanol levels are not related to coagulation changes, as measured by thromboelastography, in traumatic brain injury patients. World Neurosurg 112:e216-e222. https://doi.org/10.1016/j.wneu.2018.01.025

11. Scheyerer MJ, Dütschler J, Billeter A et al (2014) Effect of elevated serum alcohol level on the outcome of severely injured patients. Emerg Med J 31(10):813-817. https://doi.org/10.1136/ emermed-2013-202804

12. Pories SE, Gamelli RL, Vacek P, Goodwin G, Shinozaki T, Harris F (1992) Intoxication and injury. J Trauma 32(1):60-64. https:// doi.org/10.1097/00005373-199201000-00013

13. Alexander S, Kerr ME, Yonas H, Marion DW (2004) The effects of admission alcohol level on cerebral blood flow and outcomes after severe traumatic brain injury. J Neurotrauma 21(5):575-583. https://doi.org/10.1089/089771504774129900

14. Stuke L, Diaz-Arrastia R, Gentilello LM, Shafi S (2007) Effect of alcohol on Glasgow Coma Scale in head-injured patients. Ann Surg 245(4):651-655. https://doi.org/10.1097/01.sla.0000250413. 41265.d3

15. Shandro JR, Rivara FP, Wang J, Jurkovich GJ, Nathens AB, MacKenzie EJ (2009) Alcohol and risk of mortality in patients with traumatic brain injury. J Trauma 66(6):1584-1590. https://doi.org/ 10.1097/TA.0b013e318182af96

16. Cunningham RM, Maio RF, Hill EM, Zink BJ (2002) The effects of alcohol on head injury in the motor vehicle crash victim.
Alcohol Alcohol 37(3):236-240. https://doi.org/10.1093/alcalc/ 37.3.236

17. Nath FP, Beastal G, Teasdale GM (1986) Alcohol and traumatic brain damage. Injury 17(3):150-153. https://doi.org/10.1016/ 0020-1383(86)90320-7

18. Aziz H, Siordia JA, Rhee P et al (2015) Analyzing the effects of alcohol on adolescent trauma using the National Trauma Data Bank. J Trauma Acute Care Surg 79(3):463-467. https://doi.org/ 10.1097/TA.0000000000000777

19. Newgard CD, Fildes JJ, Wu L et al (2013) Methodology and Analytic Rationale for the American College of Surgeons Trauma Quality Improvement Program. J Am Coll Surg 216(1):147-157. https://doi.org/10.1016/j.jamcollsurg.2012.08.017

20. Okidi R, Ogwang DM, Okello TR et al (2020) Factors affecting mortality after traumatic brain injury in a resource-poor setting. BJS Open 4(2):320-325. https://doi.org/10.1002/bjs5.50243

21. Harrison-Felix C, Whiteneck G, DeVivo M, Hammond FM, Jha A (2004) Mortality following rehabilitation in the traumatic brain injury model systems of care. NeuroRehabilitation 19(1):45-54

22. Hicks BA, Morris JA, Bass SM, Holcomb GW, Neblett WW (1990) Alcohol and the adolescent trauma population. J Pediatr Surg 25(9):944-949. https://doi.org/10.1016/0022-3468(90) 90235-2

23. Ilie G, Adlaf EM, Mann RE et al (2014) The moderating effects of sex and age on the association between traumatic brain injury and harmful psychological correlates among adolescents. PLoS One 9(9):e108167. https://doi.org/10.1371/journal.pone.0108167

24. Raj R, Mikkonen ED, Siironen J, Hernesniemi J, Lappalainen J, Skrifvars MB (2016) Alcohol and mortality after moderate to severe traumatic brain injury: a meta-analysis of observational studies. J Neurosurg 124(6):1684-1692. https://doi.org/10.3171/ 2015.4.JNS141746

25. Woolf PD, Hamill RW, Lee LA, Cox C, McDonald JV (1987) The predictive value of catecholamines in assessing outcome in traumatic brain injury. J Neurosurg 66(6):875-882. https://doi. org/10.3171/jns.1987.66.6.0875

26. Wilde EA, Bigler ED, Gandhi PV et al (2004) Alcohol abuse and traumatic brain injury: quantitative magnetic resonance imaging and neuropsychological outcome. J Neurotrauma 21(2):137-147. https://doi.org/10.1089/089771504322778604

27. Brown SA, Tapert SF, Granholm E, Delis DC (2000) Neurocognitive functioning of adolescents: effects of protracted alcohol use. Alcohol Clin Exp Res 24(2):164-171

28. Guilhaume-Correa F, Cansler SM, Shalosky EM, Goodman MD, Evanson NK (2020) Greater neurodegeneration and behavioral deficits after single closed head traumatic brain injury in adolescent versus adult male mice. J Neurosci Res 98(3):557-570. https://doi.org/10.1002/jnr.24535

29. Rivara FP, Koepsell TD, Wang J et al (2011) Disability 3, 12, and 24 months after traumatic brain injury among children and adolescents. Pediatrics 128(5):e1129-e1138. https://doi.org/10. 1542/peds.2011-0840

Publisher's Note Springer Nature remains neutral with regard to jurisdictional claims in published maps and institutional affiliations. 